\title{
Translationally invariant treatment of pair correlations in nuclei II. Tensor correlations
}

\author{
R.F. Bishop ${ }^{a}$, R. Guardiola ${ }^{b}$, I. Moliner ${ }^{b}$, J. Navarro ${ }^{c}$, M. Portesi ${ }^{c}$, \\ A. Puente ${ }^{d}$, N.R. Walet ${ }^{\mathrm{a}, 1}$ \\ ${ }^{a}$ Department of Physics, UMIST, P.O. Box 88, Manchester M60 IQD, UK \\ b Dpto. de Física Atómica, Molecular y Nuclear, Universitat de Valencia, Avda. Dr. Moliner 50, \\ E-46100 Burjassot, Spain \\ c IFIC (Centre Mixt CSIC - Universitat de Valencia), Avda. Dr. Moliner 50, E-46100 Burjassot, Spain \\ d Departament de Física, Universitat de les Illes Balears, E-07071 Palma de Mallorca, Spain
}

Received 3 August 1998; revised 30 September 1998; accepted 1 October 1998

\begin{abstract}
We study the extension of our translationally invariant correlated-cluster treatment of few-body nuclear systems to include tensor forces and correlations. It is shown that a direct application of our method is not as successful for realistic V6 interactions as our previous results for V4 potentials suggested. We investigate the cause in detail for the case of ${ }^{4} \mathrm{He}$, and show that a combination of the method of translationally invariant configuration interactions truncated at second order with that of Jastrow-correlated wave functions seems to be a lot more powerful, thereby suggesting that for mildly to strongly repulsive forces such a hybrid procedure may be an appropriate description. (c) 1998 Elsevier Science B.V.
\end{abstract}

PACS: 21.60.-n

Keywords: Tensor correlations; Translational invariance; Jastrow correlations; V6 forces; $A \leqslant 16$

\section{Introduction}

The last few years have seen an impressive progress in the microscopic description of light, $A \lesssim 16$, nuclei directly in terms of bare realistic nucleon-nucleon interactions.

\footnotetext{
${ }^{1}$ E-mail: niels.walet@umist.ac.uk 
Among the most important developments are the variational and quantum Monte Carlo methods [1-4], the stochastic variational approach [5], the fully converged calculations within the Faddeev and Faddeev-Yakubowsky theories [6], and analogous calculations in the correlated hyperspherical harmonics expansions [7].

Some of these approaches are limited by their very nature to three- or four-body systems. On the other hand, the quantum Monte Carlo method, the stochastic variational method and the correlated hyperspherical harmonics expansions are being extended to nuclear systems with up to around 8 or 10 particles. At present the main limitations are both computer speed and computer memory, and even dealing with one additional particle requires an impressive growth of computing power. For example, Table 1 in Ref. [8] gives an indication of the computational requirements of quantum Monte Carlo calculations.

At the same time we have been trying to apply what is essentially a many-body technique (the coupled-cluster method or CCM) to light systems. When dealing with finite systems of mutually interacting particles, it is important to separate the centreof-mass (CM) motion exactly from the intrinsic motion. In the framework of the CCM it has been shown that it is possible to do so by using very special correlation operators [9]. Inspired by the CCM we have constructed a variational method that is explicitly translationally invariant, but which includes only a linearised correlation operator. This procedure, the translationally invariant configuration-interaction (TICI) method $[9,10]$, is the subject of this paper. The procedure has mainly been used in a two-body implementation, called TICI2. In recent investigations [11,12] this method has been extended to deal with saturated nuclei within the $0 p$ shell, using state-dependent (SD) pair correlations between nucleons. Calculations have been performed for various V4 nucleon-nucleon interactions.

Another way to appreciate the TICI2 method is to consider it as a special formulation of the configuration-interaction (CI) method. The main difference with conventional CI calculations lies in the way the interacting configurations are selected. In the TICI2 approach these are restricted to the most general single-pair excitations which leave the CM coordinate unaffected, and are not limited to a few major shells, as in conventional shell model approaches. These configurations are very similar to those considered in the so-called potential basis in the hyperspherical harmonics formalism [13].

For systems of bosons it has been possible to extend the TICI2 method to deal with the full second-order CCM problem [14]. In this approach, called TICC2, the ground-state wave function contains the excitation of all possible independent pairs. In the specific case of ${ }^{4} \mathrm{He}$ with Wigner forces it was found that there is no significant improvement when going from TICI2 to TICC2, indicating the lack of importance of two-pair excitations by contrast with triplet excitations. One should beware, however, that there is no guarantee that this statement holds for realistic nuclear forces with their complicated operatorial structure.

In our previous work [11] we have shown how well the TICI2 method can deal with V4 forces and V4 correlations, defined explicitly in Section 2. In this paper we shall discuss the extension to tensor forces for $0 p$ shell nuclei. The TICI 2 formalism is pre- 
sented in Section 2, together with a hybrid method that adds scalar Jastrow correlations to it. In Section 3 we present our results, with a special emphasis on the case of ${ }^{4} \mathrm{He}$ nucleus. We find that in order to get a good description of the ${ }^{4} \mathrm{He}$ nucleus we need to mix the operatorial structure of the TICI2 wave function with a scalar Jastrow-like factor. We show that this improves V4 calculations as well, but not as dramatically as in the V6 case. In Section 4 we draw some conclusions about the new hybrid method.

\section{Formalism}

\subsection{Linear pair correlations}

In the SUB2 truncation of the CCM, the ground-state wave function of an $A$-particle system is parametrised as

$$
\left|\Psi_{0}\right\rangle=\exp \left(S_{1}+S_{2}\right)|\Phi\rangle,
$$

where $|\Phi\rangle$ is an independent-particle wave function with appropriate quantum numbers, and the cluster operators $S_{1}$ and $S_{2}$ promote one and two particles, respectively, out of the orbits occupied in the reference state $|\Phi\rangle$ to otherwise empty ones. If the (uncorrelated) reference state is taken to be a Slater determinant of harmonic oscillator (HO) singleparticle wave functions, the $\mathrm{CM}$ motion can be factorised exactly. The remainder of the wave function is thus translationally invariant. If one wishes to preserve this translational invariance in the state $\left|\Psi_{0}\right\rangle$, the excitations produced by the operators $S_{1}$ and $S_{2}$ can not be chosen independently. As has been demonstrated in Ref. [9], one has to construct a combination of the two operators $S_{1}$ and $S_{2}$, denoted by $S^{(1,2)}$, which mixes both oneand two-body correlations in such a way that translational invariance is preserved.

In the TICI2 method one expands the exponential of $S^{(1,2)}$, and keeps only the linear term. This can be written most concisely in the coordinate representation. Here the TICI2 wave function takes on the very appealing form

$$
\Psi_{0}\left(\boldsymbol{r}_{1}, \ldots, \boldsymbol{r}_{A}\right)=\sum_{i<j=1}^{A} f\left(r_{i j}\right) \Phi\left(\boldsymbol{r}_{1}, \ldots, \boldsymbol{r}_{A}\right) .
$$

This form is very suitable for interactions that are given in coordinate space, such as those obtained from $N N$ phase shifts. In order to deal with realistic forces we have to allow for state dependence of the pair correlation function $f$. This is done by promoting $f$ to be an operator, and expanding it in an appropriate operatorial basis, $f\left(r_{i j}\right)=$ $\sum_{p} f^{p}\left(r_{i j}\right) \Theta_{i j}^{p}$. The basis of operators to be used is generally chosen to be consistent with that of the internucleon potential employed. For interactions with V4 structure (i.e, central, but spin-isospin dependent), the operators used are $\left\{\Theta_{i j}^{p}\right\}=\left\{1, P^{\sigma}, P^{\tau}, P^{\sigma} P^{\tau}\right\}$, where $P^{\sigma}$ and $P^{\tau}$ are the usual spin- and isospin-exchange operators, respectively. When taking into account V6 interactions, we need to use a basis which also includes both the tensor operators $\mathcal{S}_{i j}$ and $\mathcal{S}_{i j} P^{\tau}$. 
The uncorrelated reference state is a single Slater determinant for the case of spinisospin saturated systems. This can be uniquely specified by the occupied harmonic oscillator states. Rather than employing the standard $n l s$ basis, it will be convenient to work in a Cartesian basis where the states are labelled by three harmonic oscillator quantum numbers $\left(n_{x}, n_{y}, n_{z}\right)$. The closed shell nuclei ${ }^{4} \mathrm{He}$ and ${ }^{16} \mathrm{O}$ are described by fully filled $n=0$ and $n=0,1$ states, respectively; it also allows for the use of non-spherical reference states for the ${ }^{8} \mathrm{Be}$ and ${ }^{12} \mathrm{C}$ nuclei, $\left|(0,0,0)^{4}(0,0,1)^{4}\right\rangle$ and $\left|(0,0,0)^{4}(1,0,0)^{4}(0,1,0)^{4}\right\rangle$, respectively, which has proven to be better than considering a spherical reference state [11].

In the specific case of the ${ }^{4} \mathrm{He}$ nucleus, the reference wave function $|\Phi\rangle$ is fully space-symmetric and so the action of the spin-exchange operator is equal to the isospinexchange one, with opposite sign (i.e. space symmetry makes $P_{i j}^{\sigma} P_{i j}^{\tau} \equiv-1$ ). Thus in this case the basis for the correlations is overcomplete and to avoid problems when solving for the correlation functions $f^{p}$ we discard the redundant terms, and work in a basis of only three operators, $1, P^{\sigma}$ and $\mathcal{S}$.

In order to determine the ground-state energy we need to compute the expectation value of both the kinetic and the potential energy operators, as well as that of the unit matrix, to impose normalisation. The kinetic energy and norm matrices are calculated straightforwardly, and we concentrate therefore on the more complicated matrix element of the potential energy operator

$$
\langle V\rangle=\left\langle\Psi_{0}\left|\sum_{i<j} f^{\dagger}(i j) \sum_{k<l} V(k l) \sum_{m<n} f(m n)\right| \Psi_{0}\right\rangle,
$$

where the indices in parentheses identify the particles on which the corresponding operator acts. Many of the terms in the previous expression are similar, and a convenient way of dealing with them is to make a diagrammatic analysis [10] and get the topologically distinct diagrams to expand the matrix elements. The topologically distinct diagrams are schematically indicated in Fig. 1.

For each diagram we have to perform a summation over all occupied single-particle states. Since we are dealing with saturated systems, this sum is reduced to the sum over occupied harmonic oscillator states times the respective trace of the spin and isospin operators, where we must take account of all possible permutations of the labels in the ket state, as explained in more detail in [11]. Each permutation is split into a product of transpositions with space, spin and isospin parts, $(i j) \equiv\left(i^{s} j^{s}\right)\left(i^{\sigma} j^{\sigma}\right)\left(i^{\tau} j^{\tau}\right)$. The spin and isospin permutations can be represented by products of spin- and isospin-exchange operators, and are thus incorporated into the trace over spin and isospin. The final expression for matrix element related to a given diagram $d$ involving $N$ particles is

$$
\begin{aligned}
& \left\langle\Psi_{0}\left|f^{\dagger}(i j) V(k l) f(m n)\right| \Psi_{0}\right\rangle_{d} \\
& =W_{d} \sum_{p q r} \sum_{i_{1}^{s} \ldots i_{N}^{s}=1}^{A / 4} \sum_{P} \epsilon_{P} \operatorname{Tr}\left(\Theta_{i j}^{p} \Theta_{k l}^{q} \Theta_{m n}^{r} P_{P i_{1}^{\sigma} \ldots P_{N}^{\sigma}}^{\sigma} P_{P_{1}^{\tau} \ldots P_{N}^{\tau}}^{\tau}\right) \\
& \quad \times\left\langle\phi_{i_{1}^{s}}(1) \ldots \phi_{i_{N}^{s}}(N)\left|f^{p *}\left(r_{i j}\right) V^{q}\left(r_{k l}\right) f^{r}\left(r_{m n}\right)\right| \phi_{P_{1}^{s}}(1) \ldots \phi_{P i_{N}^{s}}(N)\right\rangle .
\end{aligned}
$$


2

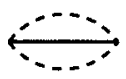

3
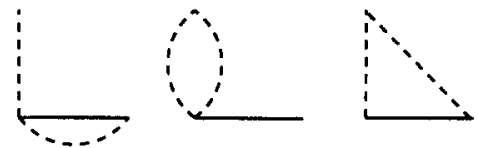

4
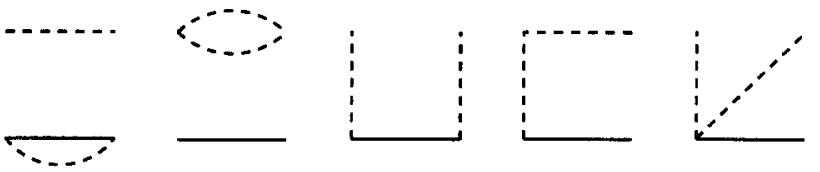

Fig. 1. A graphical representation of the diagrams contributing to the potential energy, ordered according to, and labelled by, the number of particles $N$ involved. A solid line denotes the potential, and a dashed line denotes a correlation function, one from the bra and another from the ket state. The vertices represent particles; each vertex ranges over all particles in the nucleus. If the correlation functions occupy inequivalent positions, we get two distinct contributions from each diagram. This leads to the total number of 16 contributions quoted in our previous work.

In this expression the $\phi$ 's are the spatial parts of the single-particle wave functions, $W_{d}$ is the statistical weight of the diagram and $P_{P_{1}^{\sigma} \ldots P_{N}^{\sigma}}^{\sigma}$ is the representation of the spin part of the permutation ( $P_{P_{1}^{\tau} \ldots P_{N}^{\tau}}^{\tau}$ is the same for the isospin part).

The computation of the required traces is greatly simplified if we work in the direct product representation since in this representation all the exchange and spin matrices have the same structure, with only one non-null element per row and column. In this manner the storage and matrix operations can be performed very efficiently and, although the number of required traces is large, the trace calculation may be done once and stored for later use. It is worth noting that the number of traces required in the V6 case is much higher than in the V4 case due to the complicated structure of the tensor operator.

The last step in the calculation of the matrix elements is related to the spatial integrals. There is a basic difference between the V4 and V6 cases; in the former the operatorial structure does not contribute to the spatial part of the calculation, but in the latter a spatial contribution to the integrals arises. This is due to the appearance of the spatial coordinates in the tensor operator, and we must therefore consider more types of integrals. In order to perform the integrations we expand the correlation functions in a set of Gaussians, 


$$
f^{p}(r)=\sum_{k} c_{k}^{p} \exp \left(-\beta_{k} r^{2}\right)
$$

with a factor $r^{2}$ for tensor components. The Gaussian expansion has proven to give a very accurate representation of the correlation function $f$ when both negative and positive values of $\beta$ [10] are included. In practice we use a set of around 10 Gaussians with fixed length parameters covering a sufficiently wide range of distances. The integrals are computed (see Ref. [11]) by using a recurrence relation for all integrations apart from those involving the coordinates in the potential; the remaining one-dimensional integral, which involves the potential, is then calculated numerically. For the case of ${ }^{4} \mathrm{He}$ we have also performed calculations by representing the functions $f^{p}$ on a grid in coordinate space, to check the accuracy of our results.

Since the expectation value of the Hamiltonian, as well as the norm, are bilinear in the functions $f^{p}$, we can express all expectation values as matrix elements, as obtained by substituting the expansion (5) in expressions such as (4), and isolating the contribution from a single pair of coefficients. In this way we can calculate the matrix elements of the Hamiltonian $\mathcal{H}_{m n}^{p q}$ and the norm $\mathcal{N}_{m n}^{p q}$. In terms of these matrices one obtains for the expectation value of the energy, depending on the set of coefficients $\left\{c_{m}^{p}\right\}(5)$, the formula

$$
E+E_{\mathrm{CM}}=\frac{c_{m}^{p *} \mathcal{H}_{m n}^{p q} c_{n}^{q}}{c_{m}^{p *} \mathcal{N}_{m n}^{p q} c_{n}^{q}}
$$

where summation over repeated indices must be understood both in numerator and denominator separately.

Optimising with respect to $c_{m}^{p *}$ we obtain the generalised eigenvalue problem

$$
\mathcal{H}_{m n}^{p q} c_{n}^{q}=\left(E+E_{\mathrm{CM}}\right) \mathcal{N}_{m n}^{p q} c_{n}^{q}
$$

where the lowest eigenvalue is an approximation for the ground-state energy. We must subtract the centre-of-mass energy,

$$
E_{\mathrm{CM}}=\frac{\hbar^{2}}{m} \frac{3}{4} \alpha^{2}
$$

where $\alpha$ is the inverse length HO parameter, from this eigenvalue to obtain the groundstate energy. Given that the method is strictly variational, this energy may also be optimised with respect to the harmonic oscillator parameter.

\subsection{Linear plus Jastrow correlations}

We shall also study a hybrid method which combines our TICI2 procedure with its additive state-dependent correlations, and the Jastrow method with its multiplicative state-independent correlations. This is very similar to the correlated basis functions (CBF) method as developed by Feenberg, Clark and Krotscheck [15-20] for extended systems, in which they combined Jastrow correlations and non-orthogonal perturbation theory. The method has also been applied to finite nuclei, replacing the perturbation 
expansion by a configuration-interaction approach. This specific scheme has been applied by Clark and collaborators [21,22], and by Boscá and Guardiola [23]. More recent calculations at the level of just choosing the coupling scheme have been performed by the Granada-Lecce-Pisa-Trieste collaboration $[24,25]$.

The key concept behind the CBF method is to apply a divide-and-conquer strategy to the determination of the correlated wave function. It is known that Jastrow correlations are very well suited to treat the effects of the (strongly repulsive) short-range part of the interaction, whereas a CI approach can effectively deal with medium- and longrange correlations. In our approach, we substitute the standard configuration-interaction component by our conceptually equivalent but significantly more effective TICI2 scheme. Incorporating a scalar Jastrow correlation between all pairs of nucleons in the TICI2 wave function leads to the following J-TICI2 wave function:

$$
\Psi_{J}\left(\boldsymbol{r}_{1}, \ldots, \boldsymbol{r}_{A}\right)=\left(\prod_{i<j} g\left(r_{i j}\right)\right)\left(\sum_{k<l} \sum_{p} f^{p}\left(r_{k l}\right) \Theta_{k l}^{p}\right) \Phi\left(\boldsymbol{r}_{1}, \ldots, \boldsymbol{r}_{A}\right) .
$$

Due to limitations in computer power, we have restricted the calculations to be presented below to a simplified Jastrow correlation $g(r)$ defined in terms of a single Gaussian,

$$
g(r)=1+a \mathrm{e}^{-b r^{2}} .
$$

Our wave function now depends non-linearly on three parameters, namely the harmonic oscillator parameter $\alpha$, and the width $b$ and depth $a$ of the Jastrow function. In addition, it depends linearly on the functions $f^{p}(r)$ and $f^{p}(r)^{*}$. We have to perform a variational search of the parameters to find the minimum of the expectation value $E_{J} \equiv\left\langle\Psi_{J}|H| \Psi_{J}\right\rangle /\left\langle\Psi_{J} \mid \Psi_{J}\right\rangle-E_{\mathrm{CM}}$.

\section{Results and discussion}

We shall investigate results obtained with the prescriptions defined in the previous section for several V4 and V6 interactions, and for the nuclei ${ }^{4} \mathrm{He},{ }^{8} \mathrm{Be},{ }^{12} \mathrm{C}$ and ${ }^{16} \mathrm{O}$.

We shall first look at the TICI2 method for tensor forces. Afterwards, we discuss J-TICI2 results for the specific case of ${ }^{4} \mathrm{He}$, both for V4 and V6 internucleon interactions. At the end of this section we analyse the interplay of both kinds of correlations. Preliminary versions of these results have been presented previously in conference contributions $[12,26]$.

\subsection{TICI2 results for V6 interactions}

We have performed calculations with a wide variety of interactions: the Gogny potential [27], the de Tourreil-Sprung super-soft-core potential (SSC) [28], and the Argonne potentials AV14 [29] and AV18 [30]. In all the cases we have used only the V6 part of the interactions, ignoring the terms related to the spin-orbit and other different operators. The very different character of these potentials, specially at short distances, can 

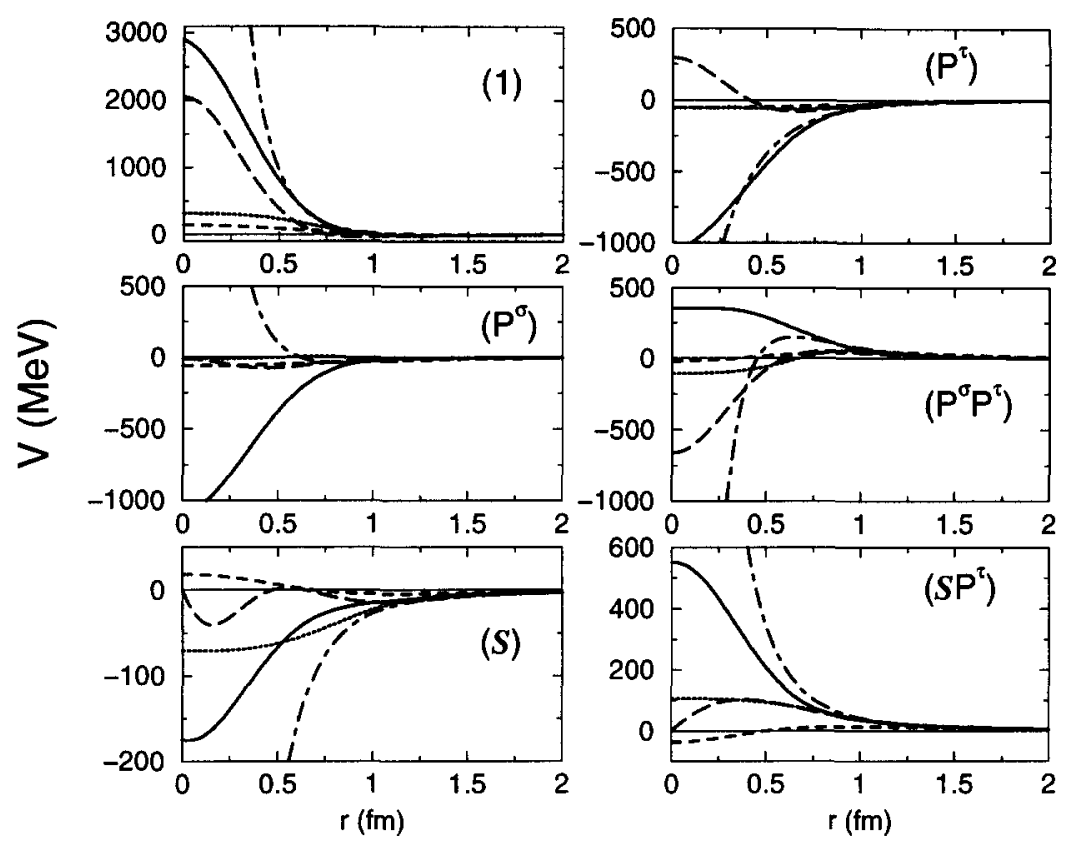

Fig. 2. A comparison between the V6 potentials used in this paper. The horizontal axis in each panel shows $r$ (in fm), the vertical axis the potential strength (in $\mathrm{MeV}$ ). Each panel represents one operatorial channel, for each of the operators used to expand the correlation functions, as indicated in the figure. The solid line is AV14, the long-dashed line is AV18. The dotted line is the SSC, and the medium dashed line is the Gogny. The short-long dashed line is the Reid-V6 potential.

be seen in Fig. 2. Here we include, in addition to the potentials mentioned above, a modified version of the Reid soft-core potential [31,32] (called Reid-V6), used in later calculations for ${ }^{4} \mathrm{He}$ only, and discussed further in Subsection 3.3.

The introduction of tensor correlations in our scheme increases dramatically the computer time needed in the calculations, as stated in Section 2, and it has taken considerable effort to deal with the case of ${ }^{16} \mathrm{O}$. In Table 1 we give the results for the ground-state binding energy obtained for the interactions mentioned above. We also list the value of the harmonic oscillator parameter corresponding to the minimum. Using our method, we find that the V6 parts of the AV14 and AV18 potentials give a very low value for the ${ }^{4} \mathrm{He}$ binding energy. The nuclei ${ }^{8} \mathrm{Be},{ }^{12} \mathrm{C}$ and ${ }^{16} \mathrm{O}$ are even less bound, which is not what we expect to see. The results obtained for the SSC and especially the Gogny potential exhibit a more acceptable behaviour, showing at least a monotonic increase in binding energy for increasing values of $A$.

These results are very surprising in the light of the quality of our previous results for V4 forces [11], and merit further investigation. One extremely important difference is the competition between central and tensor forces. In the V4 forces used in our previous work most of the binding originates in the central channel. For all of the V6 forces used here, most binding is obtained from the off-diagonal tensor force, i.e. the matrix element between the central and tensor-correlated channel. This matrix element competes with a 
Table 1

Binding energies (in MeV) of $0 p$-shell nuclei using TICI2 V6-like correlations, with the V6 part of the Gogny, SSC, AV14 and AV18 interactions. The parameter $\alpha$ is the optimal harmonic oscillator parameter (in $\mathrm{fm}^{-1}$ )

\begin{tabular}{llll}
\hline Interaction & Nucleus & $-E$ & $\alpha$ \\
\hline Gogny/V6 & ${ }^{4} \mathrm{He}$ & 27.36 & 0.70 \\
& ${ }^{8} \mathrm{Be}$ & 40.79 & 0.58 \\
& ${ }^{12} \mathrm{C}$ & 73.37 & 0.61 \\
& ${ }^{16} \mathrm{O}$ & 128.68 & 0.64 \\
SSC/V6 & ${ }^{4} \mathrm{He}$ & 24.12 & 0.68 \\
& ${ }^{8} \mathrm{Be}$ & 25.98 & 0.54 \\
& ${ }^{12} \mathrm{C}$ & 39.64 & 0.54 \\
& ${ }^{16} \mathrm{O}$ & 63.55 & 0.55 \\
AV14/V6 & ${ }^{4} \mathrm{He}$ & 14.77 & 0.59 \\
& ${ }^{8} \mathrm{Be}$ & 9.26 & 0.43 \\
& ${ }^{12} \mathrm{C}$ & 10.50 & 0.41 \\
& ${ }^{16} \mathrm{O}$ & 14.97 & 0.40 \\
& ${ }^{4} \mathrm{He}$ & 15.40 & 0.61 \\
& ${ }^{8} \mathrm{Be}$ & 11.13 & 0.47 \\
& ${ }^{12} \mathrm{C}$ & 14.96 & 0.46 \\
& ${ }^{16} \mathrm{O}$ & 23.76 & 0.46 \\
\hline
\end{tabular}

strong repulsion in the central channel. From Table 1 and Fig. 2 we see that the origin of our problem seems to be related to the size of the repulsive core of the potential. We get the most sensible result for the weak Gogny force, a slightly worse result for the more repulsive SSC potential, and very poor results for the AV14 and AV18 interactions.

\subsection{J-TICI2 results for $V 4$ interactions}

In order to shed some more light on these results we have investigated the ${ }^{4} \mathrm{He}$ system more deeply. For this nucleus we can make comparisons with other variational calculations and with Green function Monte Carlo results.

We have performed calculations in the ${ }^{4} \mathrm{He}$ system by using the J-TICI2 wave function of Eq. (9). With this ansatz, as already outlined in Section 2, we keep the operatorial structure of TICI2 but add the flexibility of the Jastrow correlations.

In order to investigate the origin of the different quality of TICI2 calculations for V4 and V6 interactions, we will start by considering the simpler case of the V4 potentials that were studied in our previous paper [11], namely the Brink-Boeker B1 [33], the Afnan-Tang S3 [34] and its modified version MS3 [35], and the Malfliet-Tjon MT I/III and MT V [36] potentials. In Table 2, we compare our J-TICI2 results for V4 interactions with the TICI2 ones [11], and with those from a recent Jastrow calculation [37] using state-dependent correlations. In the case of the purely Wignerlike interactions, we also quote some diffusion Monte Carlo (DMC) results [38] which provide a benchmark for the calculations. 
Table 2

Comparison between different results for the ${ }^{4} \mathrm{He}$ binding energy (in MeV) using several V4 potentials. $S 3_{\mathrm{W}}$ is the Wigner part of the $\mathbf{S 3}$ potential. Jastrow results refer to calculations using state-dependent (SD) correlations containing two Gaussians

\begin{tabular}{lllll}
\hline & TICI2 $[11]$ & J-TICI2 & SD-Jastrow [37] & DMC [38] \\
\hline B1 & 37.86 & 38.28 & $38.27[38]$ & $38.32 \pm 0.01$ \\
S3 & 28.19 & 30.16 & 29.94 & \\
S3W & 25.41 & 27.20 & 27.21 & $27.35 \pm 0.02$ \\
MS3 & 27.99 & 29.97 & 29.70 & $31.32 \pm 0.02$ \\
MT V & 29.45 & 31.21 & 30.88 & \\
MT I/III & 30.81 & 32.70 & 32.01 & \\
\hline
\end{tabular}

The main difference between the J-TICI2 and the SD-Jastrow calculations lies in the way the state dependence of the correlations is parametrised. In the case of SDJastrow, the correlation factor is assumed to have the same operatorial structure as the potential, and the product of correlated pairs $\prod_{i<j}\left(\sum_{p} g^{p}\left(r_{i j}\right) \Theta_{i j}^{p}\right)$ must be appropriately symmetrised to maintain the Fermi statistics. In the SD-Jastrow results quoted in Table 2, each of the functions $g^{p}(r)$ was parametrised as the sum of two Gaussians. On the other hand, in the J-TICI2 calculation the Jastrow correlation factor is state-independent, so that all of the operatorial dependence is contained in the pair correlation $f(r)$. For computational reasons, the Jastrow factor in the latter case was parametrised by a single Gaussian, as mentioned at the end of the previous section.

In these model calculations we are trying to assess the effect of superimposing the scalar Jastrow correlation on top of the linear pair correlations. We see from Table 2 that inclusion of the scalar Jastrow factor lowers the ground-state energy (i.e. increases the binding energy) of the pure TICI2 results by around 1-2 MeV. The binding energies obtained for the Wigner-like potentials B1 and $\mathrm{S} 3_{\mathrm{W}}$ from the J-TICI2 method and from the use of SD-Jastrow correlations are practically the same, but the former works better for more realistic interactions with operatorial structure. Especially interesting is the case of the MT potentials: for the MT V form, the J-TICI2 value is already better than the SD-Jastrow one, despite the fact that the former includes only one Gaussian whereas the latter includes two Gaussians in the correlation functions $g$. This improvement can be explained by realizing that the MT potentials contain a very short-range Yukawa core. The optimal width of the Gaussian in the Jastrow correlation is very small $\left(b \sim 5 \mathrm{fm}^{-2}\right)$, which cannot provide at the same time a good description for the long-range part of the correlation required to modify the independent-particle reference state. We also observe the proximity of the J-TICI2 and DMC results, and we hence conclude that, for these interactions, the J-TICI2 approach gives an excellent way to treat the ground state of ${ }^{4} \mathrm{He}$.

In conclusion, the $\mathrm{V} 4$ results indicate the relevance of the hybrid J-TICI2 description, assigning to each part of the wave function its proper role: the accurate description of the short-range behaviour is assigned to the scalar Jastrow factor, whereas the TICI2 part is responsible for the medium-range correlations. 


\subsection{J-TICI2 results for $V 6$ interactions}

This section deals with the most relevant application of the J-TICI2 ansatz, namely the consideration of realistic V6 forces. In addition to the potentials used before we have also considered a modified version of the original channel-dependent Reid softcore (RSC) interaction [31], which presents a much harder core than the previous ones, and thus emphasises the role of short-range correlations. Please note that we use the same channel-independent Reid-V6 version of the RSC potential as has been used in the GFMC calculations of Ref. [32].

Table 3 summarises our V6 results. In addition to the results obtained with the TICI2 and J-TICI2 descriptions, the table also includes calculations with a V6 state-dependent Jastrow correlation computed with the variational Monte Carlo (VMC) method, as well as the V6 contribution to the energy computed with the Green function Monte Carlo (GFMC) method for two interactions, AV14 [39] and Reid-V6 [32], which provide the benchmark results. Our state-dependent Jastrow VMC calculations have been carried out by using a separate Jastrow correlation function obtained by solving Schrödinger-like equations for each operatorial channel, as in Ref. [40].

The pattern of V6 results is rather different from the V4 cases. The effect of the inclusion of the state-independent Jastrow correlations on top of the state-dependent TICI2 pair correlations is most impressive for strongly repulsive potentials, such as the Reid-V6 [31], in which the gain in binding energy is about $17 \mathrm{MeV}$. Clearly, with the exception of the extremely soft Gogny potential, the J-TICI2 method has not converged to the level of accuracy of the VMC (let alone GFMC) results.

We note, however, that one degree of freedom, which has not yet been explored due to computational limitations, is the use of a more general parametrisation for the Jastrow correlation function $g$ in the J-TICI2 calculation in terms of multiple Gaussians. The effect of adding more Gaussian components into the parametrisation of Jastrow correlation factors has been studied rather carefully in previous work $[37,38]$ of some of the present authors. For example, we showed [38] that for the ${ }^{4} \mathrm{He}$ nucleus, a simple (state-independent) Jastrow calculation with two or more optimised Gaussian components yielded approximately $2 \mathrm{MeV}$ more binding for the B1, S3 and MT V potentials than in the case with a single optimised Gaussian. A similar (or greater)

Table 3

Same as Table 2, but referring to the V6 parts of several realistic potentials. The result attributed to Ref. [32] was obtained by adding the expectation values of the kinetic energy and the V6 part of the potential cited in that paper, and hence it is not a fully optimised result

\begin{tabular}{lclll}
\hline & TICl2 $[12]$ & J-TICI2 & VMC [12] & GFMC \\
\hline Gogny/V6 & 27.36 & 27.58 & $27.71 \pm 0.06$ & \\
SSC/V6 & 24.12 & 26.74 & $29.20 \pm 0.12$ & \\
AV14/V6 & 14.77 & 20.37 & $23.24 \pm 0.08$ & $24.79 \pm 0.20[39]$ \\
AV18/V6 & 15.40 & 21.08 & $24.80 \pm 0.09$ & \\
Reid-V6 & 5.67 & 22.70 & $27.82 \pm 0.12$ & $28.30 \pm 0.12[32]$ \\
\hline
\end{tabular}


degradation of the VMC results shown in Table 3 would be expected if these were to be replaced with Jastrow correlation functions optimised at the single Gaussian level.

We expect some comparable improvement in energy, in the J-TICI2 results when the Jastrow correlation function is parametrised in terms of more than one Gaussian component. However, it is as yet unclear whether this will provide us with the bulk of the missing binding. The nature of the binding (which, as we shall see below, is mainly through off-diagonal components of the tensor force) is such that we might expect that iterations of the tensor correlations could also be important. The relevance of such effects would only be addressed in a full TICC2 calculation.

\subsection{Analysis of the results}

In this section we present our thoughts about the origins of the different behaviour of the TICI 2 and the J-TICI 2 results. They are based on the analysis of the overall size of the nucleus and on a comparison of the contributions of the various operatorial parts to the energy.

The first way of analysing the results is to compare the values of the harmonic oscillator parameter as shown in Table 4. We see that, even for V4 interactions, it is the increase in $\alpha$, that is provided by the inclusion of the Jastrow correlation factors in Eq. (9), which drives the improvements of the energy. This need not surprise us; with a Jastrow correlation to kill off the short-range repulsion, we can use the additive CI2 correlations to get the long-range physics right. Without Jastrow correlations the CI 2 correlation needs to play both roles, which works better at smaller $\alpha$, where the nucleons are automatically further apart. The value of the HO parameter $\alpha_{J}$ for the J-

Table 4

TICI2 results $(E, \alpha)$ and J-TICI2 results $\left(E_{J}, \alpha J, a, b\right)$ for ${ }^{4} \mathrm{He}$, using different interactions with V4 and V6 structures. Binding energies are given in $\mathrm{MeV}$, the HO parameters $\alpha$ and $\alpha_{J}$ in $\mathrm{fm}^{-1}$, the depth $a$ of the J-TICI2 Gaussian correlation function of Eq. (10) is dimensionless, and its width $b$ is measured in $\mathrm{fm}^{-2}$

\begin{tabular}{|c|c|c|c|c|c|c|}
\hline & \multicolumn{2}{|c|}{ TICI2 } & \multicolumn{4}{|c|}{ J-TICI2 } \\
\hline & $-E$ & $\alpha$ & $-E_{J}$ & $\alpha_{J}$ & $a$ & $b$ \\
\hline \multicolumn{7}{|c|}{ V4 Interactions } \\
\hline B1 & 37.86 & 0.73 & 38.28 & 0.77 & -0.41 & 1.8 \\
\hline S3 & 28.19 & 0.72 & 30.16 & 0.78 & -0.70 & 2.1 \\
\hline$s 3_{W}$ & 25.41 & 0.72 & 27.20 & 0.74 & -0.69 & 2.3 \\
\hline MS3 & 27.99 & 0.71 & 29.97 & 0.74 & -0.70 & 2.1 \\
\hline MT V & 29.45 & 0.74 & 31.21 & 0.75 & -0.87 & 5.6 \\
\hline MT I/III & 30.81 & 0.74 & 32.70 & 0.75 & -0.88 & 5.0 \\
\hline \multicolumn{7}{|c|}{ V6 Interactions } \\
\hline Gogny/V6 & 27.36 & 0.70 & 27.58 & 0.72 & -0.29 & 1.9 \\
\hline SSC/V6 & 24.12 & 0.68 & 26.74 & 0.75 & -0.66 & 2.1 \\
\hline AV14/V6 & 14.77 & 0.59 & 20.37 & 0.71 & -0.93 & 2.7 \\
\hline AV18/V6 & 15.40 & 0.61 & 21.08 & 0.74 & -0.92 & 3.2 \\
\hline Reid-V6 & 5.67 & 0.43 & 22.70 & 0.72 & -1.05 & 2.4 \\
\hline
\end{tabular}




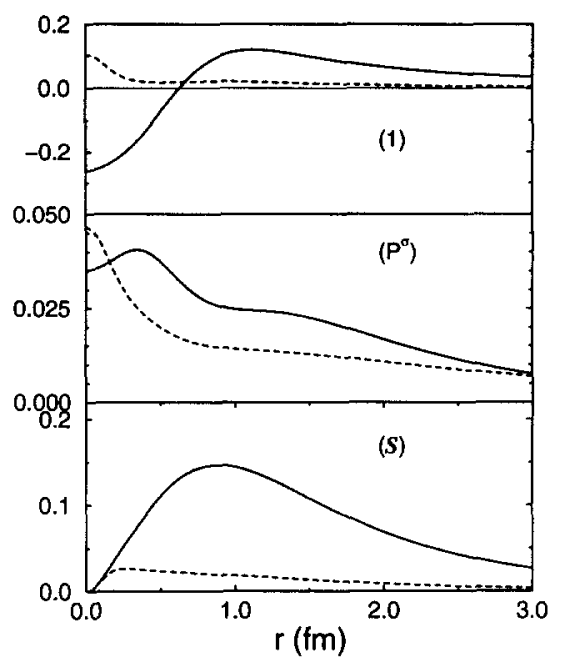

Fig. 3. A comparison between the TICl2 (solid line) and J-TICI2 (dashed line) correlation functions $f^{p}$, multiplied with $\exp \left(-\alpha^{2} r^{2} / 8\right)$, for ${ }^{4} \mathrm{He}$ obtained from a solution to the generalised eigenvalue problem (7). We have chosen the V6 part of the AV14 potential as an example, and only plot the range for $r$ from 0 to 3 $\mathrm{fm}$. The normalisation of each of the correlation functions is as in the normalised ground-state eigenfunction, but we present separate results for each of the three operatorial channels.

TICI2 ansatz does not change very much when considering different interactions, ranging between 0.71 and $0.78 \mathrm{fm}^{-1}$ and thus leads to similar overall sizes for the system. By contrast, the values of the Jastrow parameters present a wider range of variation, both in depth and in range, mainly due to the fact that the short-range behaviour of the potentials is very different from one to another.

In Fig. 3 we compare the resulting correlation functions $f^{p}$ for the AV14/V6 potential. Note that all these functions grow exponentially at large radii, but these contributions are moderated by the harmonic oscillator wave functions. For that reason we have multiplied these correlation function with the factor $\exp \left[-(1 / 2 A) \alpha^{2} r^{2}\right]$, arising from the relativecoordinate part of the harmonic oscillator Slater determinant [11]. Sizes are related to the norm of the related component in the ground-state eigenfunction. As expected we see most change for the central correlation function. This has a node for the case of the TICI 2 calculation, due to the fact that it tries to screen part of the hard-core contribution. For the J-TICI 2 method this part of the correlation function is very smooth and structureless, apart from a small bump at small distances. We see that the tensor correlations are again rather smooth functions, which lends support to our suggestion to include only state-independent Jastrow-factors in our J-TICI2 method.

A slightly deeper understanding of the mechanism of binding can be obtained by decomposing the Hamiltonian in channels, where in each channel we use the combination of functions that was obtained from the ground-state diagonalisation. This effectively reduces all matrices to three-by-three ones (one index for each of the central, spindependent and tensor-correlated channels) which allows for easier analysis. In a more mathematical notation, 


$$
\begin{aligned}
& H_{p q}=\left\langle\Phi^{\prime}\left|\sum_{i<j} \Theta_{i j}^{p} f^{p *}\left(r_{i j}\right) H \sum_{k<l} \Theta_{k l}^{q} f^{q}\left(r_{k l}\right)\right| \Phi^{\prime}\right\rangle \equiv \sum_{m, n} c_{m}^{p *} \mathcal{H}_{m n}^{p q} c_{n}^{q}, \\
& N_{p q}=\left\langle\Phi^{\prime}\left|\sum_{i<j} \Theta_{i j}^{p} f^{p *}\left(r_{i j}\right) \sum_{k<l} \Theta_{k l}^{q} f^{q}\left(r_{k l}\right)\right| \Phi^{\prime}\right\rangle \equiv \sum_{m, n} c_{m}^{p *} \mathcal{N}_{m n}^{p q} c_{n}^{q},
\end{aligned}
$$

where $\Phi^{\prime}$ is either the model state $\Phi$ itself or the Jastrow-correlated model state, $\Phi^{\prime}\left(r_{1}, \ldots, r_{A}\right)=\prod_{i<j} g\left(r_{i j}\right) \Phi\left(r_{1}, \ldots, r_{A}\right)$, in the cases of the TICI2 or J-TICI2 calculations, respectively. The labels $p$ and $q$ run from one to three over central (1), spin $\left(P^{\sigma}\right)$ and tensor $(\mathcal{S})$, respectively.

Since the norm matrix $N$ is diagonal, we can trivially take the square root, perform the Löwdin transformation, and obtain the symmetric energy matrix,

$$
\mathcal{E}=N^{-1 / 2} H N^{-1 / 2}
$$

Let us compare these matrices in the case of the AV14 potential. For the TICI2 wave function the energy matrix, subtracting $E_{\mathrm{CM}}$ from the diagonal elements, has the value

$$
\mathcal{E}_{\mathrm{TICI} 2}=\left(\begin{array}{rrr}
12.69 & 11.94 & -96.38 \\
11.94 & 212.54 & -68.09 \\
-96.38 & -68.09 & 326.56
\end{array}\right) \text {. }
$$

The eigenvector for the ground state is $(0.960,0.033,0.278)$ with an eigenvalue of $-14.77 \mathrm{MeV}$. Once we add the Jastrow correlation we find

$$
\mathcal{E}_{\mathrm{J}-\mathrm{TICI} 2}=\left(\begin{array}{rrr}
16.35 & 15.01 & -109.66 \\
15.01 & 294.05 & -74.89 \\
-109.66 & -74.89 & 310.06
\end{array}\right) \text {. }
$$

The eigenvector for the ground state is now $(0.946,0.031,0.321)$ with eigenvalue $-20.37 \mathrm{MeV}$.

In both cases the amplitude of the spin component is very small, so that the influence of the spin channel is not of much relevance. On the other hand, both diagonal matrix elements related to the central channel and to the tensor channel are positive, so that the central-tensor coupling is the real source of negative energy.

\section{Conclusions}

We have shown that the extension of our state-dependent TICI2 calculations to deal with tensor forces and tensor correlations is not as straightforward as our previous work suggests. The reason seems to lie mainly in the competition between the shortrange repulsion induced by the central force and the long-range correlations induced by the tensor force. The presence of unscreened potential contributions, as shown in Fig. 1, makes the TICI2 method not very well suited to deal with realistic interactions. One might well argue that a full CCM calculation, where only connected diagrams appear, would alleviate this problem. Unfortunately, even though some progress has been 
made [14], a coordinate-space version of the translationally invariant CCM method has not yet been completely and consistently formulated.

Even if we could perform such a calculation, we would still not obtain full screening. Indeed, the method of Jastrow correlations is much better suited for such purposes. The argument about the source of repulsion (mainly of a central character) suggests that the J-TICI2 method proposed here should be an excellent starting point for describing light nuclei with realistic interactions. Our results for ${ }^{4} \mathrm{He}$ still give a few $\mathrm{MeV}$ less binding than the much more elaborate VMC calculations and the (statistically) exact GFMC calculations.

One element that has not yet been fully explored is a better parametrisation for the Jastrow function, and definite conclusions about the quality of our approach must be delayed until we are able to deal with a more general central Jastrow correlation. We are also actively investigating the best way to extend the J-TICI2 calculations to heavier systems.

As a last comment, we note the conceptual similarity between our approach and the correlated basis functions ( $\mathrm{CBF}$ ) description of many-body systems, which may provide another avenue for future development of hybrid methods. Indeed, this conceptual similarity with the CBF technique encourages us to believe that the J-TICI2 method will itself prove practicable in applications to both heavier nuclei and deformed or other exotic nuclei, since the CBF method has itself already been applied to medium-heavy nuclei. In the same vein we note that the extension of the J-TICI2 method to include interactions and correlations more complicated than V6 type (i.e. by the inclusion of spin-orbit and other components) presents no fundamental challenges. We are actively pursuing such extensions.

\section{Acknowledgements}

This work is supported by the Commission of the European Community under research contract No. ERBCHRXCT940456. We acknowledge support by DGICyT (Spain) under contract No. PB92-0820 (R.G., J.N. and I.M.) and contract No. PB95-0492 (A.P.). R.F.B. and N.R.W. acknowledge support of a research grant (GR/L22331) from the Engineering and Physical Sciences Research Council (EPSRC) of Great Britain.

\section{References}

[1] B.S. Pudliner, V.R. Pandharipande, J. Carlson and R.B. Wiringa, Phys. Rev. Lett. 74 (1995) 4396.

[2] B.S. Pudliner, V.R. Pandharipande, J. Carlson, S.C. Pieper and R.B. Wiringa, Phys. Rev. C 56 (1997) 1720.

[3] J. Carlson and R. Guardiola, ed., Few-Body Problems in Physics '95, Few-Body Systems Suppl. 8 (1995) 32.

[4] S.C. Pieper, R.B. Wiringa and V.R. Pandharipande, Phys. Rev. C 46 (1992) 1741.

[5] K. Varga and Y. Suzuki, Phys. Rev. C 52 (1995) 2885; Phys. Rev. A 53 (1996) 1907.

[6] W. Glöckle, H. Kamada, H. Witała, D. Hüber, J. Golak, K. Miyagawa and S. Ishikawa, in: Few-Body Problems in Physics '95, ed. R. Guardiola, Few-Body Systems Suppl. 8 (1995) 9, and references therein. 
[7] A. Kievsky, M. Viviani and S. Rosati, Nucl. Phys. A 551 (1993) 241; A 577 (1994) 511; S. Rosati, M. Viviani and A. Kievsky, in: Few-Body Problems in Physics '95, ed. R. Guardiola, FewBody Systems Suppl. 8 (1995) 21.

[8] S.C. Pieper, Microscopic Quantum Many-Body Theories and Their Applications, ed. J. Navarro and A. Polls, Lecture Notes Phys. 510 (Springer, Berlin, 1998) p. 337.

[9] R.F. Bishop, M.F. Flynn, M.C. Boscá, E. Buendía and R. Guardiola, Phys. Rev. C 42 (1990) 1341; J. Phys. G: Nucl. Part. Phys. 16 (1990) L61; Condensed Matter Theories, Vol. 5, ed. V.C. Aguilera-Navarro (Plenum, New York, 1990) p. 253.

[10] R.F. Bishop, E. Buendía, M.F. Flynn and R. Guardiola, Condensed Matter Theories, Vol. 6, ed. S. Fantoni and S. Rosati (Plenum, New York, 1991) p. 405; J. Phys. G: Nucl. Part. Phys. 17 (1991) 857; 18 (1992) 1157; 19 (1993) 1163.

[11] R. Guardiola, P.I. Moliner, J. Navarro, R.F. Bishop, A. Puente and Niels R. Walet, Nucl. Phys. A 609 (1996) 218.

[12] I. Moliner, R. Guardiola, J. Navarro, R.F. Bishop, Niels R. Walet and A. Puente, Condensed Matter Theories, Vol. 13, ed. J. da Providencia and R.F. Bishop (Nova Science, New York, 1998), in press.

[13] R. Guardiola, P.I. Moliner and J. Navarro, Phys. Lett. B 383 (1996) 243.

[14] R. Guardiola, I. Moliner, J. Navarro and M. Portesi, Nucl. Phys. A 628 (1998) 187.

[15] E. Feenberg and J.W. Clark, Phys. Rev. 113 (1959) 388.

[16] E. Feenberg and C.W. Woo, Phys. Rev. 137 (1965) 391.

[17] E. Feenberg, Theory of quantum fluids (Academic Press, New York, 1969).

[18] J.W. Clark and P. Westhaus, Phys. Rev. 141 (1966) 833; 149 (1966) 990.

[19] J.W. Clark, in: Progress in Particle and Nuclear Physics, Vol. 2, ed. D.H. Wilkinson (Pergamon, Oxford, 1979).

[20] E. Krotscheck and J.W. Clark, Nucl. Phys. A 328 (1979) 73.

[21] M.L. Ristig, W.J. Ter Louw and J.W. Clark, Phys. Rev. C 3 (1971) 1504.

[22] L.R. Mead and J.W. Clark, Phys. Lett. B 90 (1980) 331.

[23] M.C. Boscá and R. Guardiola, Nucl. Phys. A 476 (1988) 471.

[24] G. Có, A. Fabrocini and S. Fantoni, Nucl. Phys. A 549 (1992) 439; A 568 (1994) 73.

[25] F. Arias de Saavedra, G. Có, A. Fabrocini and S. Fantoni, Nucl. Phys. A 605 (1996) 359.

[26] M. Portesi, J. Navarro, R. Guardiola, I. Moliner, R.F. Bishop and N.R. Walet, Jastrow-correlated configuration-interaction description of light nuclei, Proc. 16th Euro. Conf. on Few-Body Problems in Physics, Autrans (France, June 1998). To be published in Few-Body Syst. Suppl.

[27] D. Gogny, P. Pires and R. de Tourreil, Phys. Lett. B 32 (1970) 591.

[28] R. de Tourreil and D.W.L. Sprung, Nucl. Phys. A 201 (1973) 193.

[29] R.B. Wiringa, R.A. Smith and T.L. Ainsworth, Phys. Rev, C 29 (1984) 1207.

[30] R.B. Wiringa, V.G.J. Stoks and R. Schiavilla, Phys. Rev. C 51 (1995) 38.

[31] R.V. Reid, Ann. Phys. (NY) 50 (1968) 411.

[32] J. Carlson, Phys. Rev. C 38 ( 1988) 1879.

[33] D.M. Brink and E. Boeker, Nucl. Phys. A 91 (1967) 1.

[34] I.R. Afnan and Y.C. Tang, Phys. Rev. 175 (1968) 1337.

[35] R. Guardiola, A. Faessler, H. Müther and A. Polls, Nucl. Phys. A 371 (1981) 79.

[36] R.A. Malfliet and J.A. Tjon, Nucl. Phys. A 127 (1969) 161.

[37] R. Guardiola and M. Portesi, J. Phys. G: Nucl. Part. Phys. 24 (1998) L37.

[38] R.F. Bishop, E. Buendía, M.F. Flynn and R. Guardiola, J. Phys. G: Nucl. Part. Phys. 18 (1992) L21

[39] J. Carlson, Phys. Rev. C 36 (1987) 2026.

[40] J.A. Carlson and R.B. Wiringa, Computational Nuclear Physics 1, ed. K. Langanke, J.A. Maruhn and S.E. Koonin (Springer, Berlin, 1991). 